Fecha de recepción: abril 2020

Fecha de aceptación: mayo 2020

Versión final: junio 2020

\section{¿Institución de Órdenes Alternativos o Construcción de Dinámicas Plurales? La dimensión del espacio local en los procesos de significación local/global}

Elisa Edith Luna ${ }^{(1)}$

\begin{abstract}
Resumen: Interesa resaltar la caracterización histórica del arte como elemento político disruptivo. Y es, observando que dicho proceso solo ha sido posible desde una articulación consciente y crítica de los espacios locales y sus caracterizaciones, que el presente trabajo, se sitúa en la búsqueda de producciones alternativas a los órdenes establecidos. Viendo su legitimación en la representación de las articulaciones negadas por el espacio local. Este objetivo se trabaja, a través del análisis de las producciones de sentido de las Organizaciones Civiles Ambientales de la Provincia de San Juan, entendiendo a dicho actor como representante de estas articulaciones. Pudiendo identificar en sus regímenes de visibilidad, una etapa reproductiva que, plantea en la propia existencia de estas organizaciones y en sus procesos de reproducción de sentidos una propuesta económica, estética y política alternativa local, es decir, una propuesta conceptual que considera un orden plural, como representación de la necesidad de establecer nuevas tipologías de espacio que visibilicen la intrínseca articulación entre lo público y lo privado dada en el proceso integral de producción de sentido local. Como orden emergente, basado en una perspectiva ecosistémica de lo local articulada, en forma interlocal, dentro de las lógicas del organismo global.
\end{abstract}

Palabras clave: Local - Ecosistema - Visibilidad - Estética - Comunicación.

[Resúmenes en inglés y portugués en la página 157]

(1) Diseñadora Gráfica egresada de la Universidad Nacional de San Juan, Doctoranda del Doctorado en Comunicación de la Facultad de Periodismo y Comunicación Social perteneciente a la Universidad Nacional de La Plata, Becaria CONICET en el Instituto Regional de Planeamiento y Hábitat de la Facultad de Arquitectura, Urbanismo y Diseño perteneciente a la Universidad Nacional de San Juan.

Comenzaremos identificando al soporte material de los discursos, no solo como medio, sino como parte integral de la construcción de un espacio de significación local. Ya que, entendido el soporte discursivo de esta forma, se puede observar al proceso de significación, como un proceso dialógico entre los diferentes niveles discursivos. 
Perspectiva que nos permite separar el arte de la idea de su función histórica de obra discursiva representativa de la condensación de sentidos particulares para entenderlo como parte de un espacio social local dado en articulaciones discursivas, tanto en la producción como en la reproducción de los sentidos implicados en sus prácticas.

Desde esta perspectiva dialógica entre los diferentes niveles discursivos, es que observamos, existen procesos dialógicos configurados socialmente, y procesos dialógicos normados (muchas veces a partir de estas configuraciones), basados en las propias narrativas históricas de los territorios que construyen la idea de "lugar". Entendido el mismo, como elemento articulador espacial que no solo legitima en su propia existencia significaciones sino también los modos de significar. Como también legitima las caracterizaciones y jerarquías de los elementos discursivos y, de esta forma, su implicación en los procesos de participación en la construcción de dicho espacio.

Por lo que, desde esta perspectiva, es necesario observar que el espacio local, es innegablemente, un espacio de diálogo y en consecuencia de producción de significaciones y por tanto un espacio dinámico, donde todo actor es parte de dicho diálogo (en sus diferentes formas de habitar este espacio). Observando como esto convierte al mismo en un espacio caracterizado y construido en la identificación de la pluralidad de las partes que lo integran. Es decir, observando en este hecho de reconocimiento e integración su construcción identitaria y en la negación de alguna de sus partes, la exclusión y la deconstrucción de dicha identidad.

Observando así también, en estas situaciones de negación y exclusión, a través de la deconstrucción de significaciones locales, el origen de significaciones construidas, configuradas y hasta en algunas ocasiones normadas (en las formas de reproducción discursiva) históricamente como disruptivas.

En el presente trabajo se busca analizar las mismas desde la categoría conceptual de las significaciones emergentes e identificar las posibilidades instituyentes que estas habilitan. Entendiendo esta concepción de lo emergente relacionada con la "emergencia" presente en aquellas prácticas sociales configuradas o normadas desde la negación y la exclusión de las significaciones que las mismas producen. Analizando así la emergencia de visibilidad de las dinámicas y diálogos que se generan en el espacio local, como nuevos y emergentes procesos de significación.

Es por esto que en el presente trabajo nos interesa observar la relación que guardan estas significaciones emergentes con los procesos históricamente "disruptivos" que ha presentado el arte. Entendiendo que esta tarea resulta inseparable del cuestionamiento sobre los quienes se han hallado detrás de estos procesos que han caracterizado dicha dimensión "disruptiva". Tarea también inseparable del cuestionamiento por las condiciones de producción de los discursos de estos actores, las cuales constituyen estos procesos de significación como espacio-temporalmente emergentes.

Para llevar adelante este análisis emplearemos un proceso inverso, no preguntándonos primero donde se encuentra espacio-temporalmente el arte disruptivo para luego identificar a sus actores y sus procesos de significación emergente. Sino primero donde se hallan los actores que representan en sus procesos dialógicos dichas prácticas discursivas "disruptivas" o emergentes a las cuales suele referir el arte. Para desde este lugar analizar a que elementos dialógicos espaciales responden dichas prácticas y poder cuestionarnos 
sobre los elementos estéticos que las caracterizan. Haciendo énfasis en las condiciones que presentan estos elementos tanto en la producción de sentido discursivo como en su circulación y reproducción, como una forma de analizar, no solo los posibles nuevos actores que puede presentar la practica artística contemporánea, sino también las nuevas o no tan nuevas condiciones político-sociales que la implican.

Siguiendo este camino se observa como a partir de las nuevas tecnologías de la información, ha aumentado la presencia, protagonismo y participación de las organizaciones civiles: ONGs, asambleas, cooperativas, etc. en la producción de significaciones tanto locales como interlocales, desde la "emergencia" de visibilidad de las significaciones construidas en las prácticas locales, principalmente en respuesta a las nuevas formas de organización caracterizadas y legitimadas como "globales".

Tomando, en este trabajo, por la pertinencia que da a los debates entre las producciones de significación local y las producciones de significación "global" y las condiciones espaciotemporales que sostienen estos soportes discursivos, a los protagonistas del debate ambiental. Los cuales han contribuido, con sus experiencias locales y prácticas discursivas, a una representación no solo de la emergencia de la visibilidad de las prácticas locales que los implican, sino la emergencia de una concepción de análisis que resignifique dichas prácticas desde sus posibilidades emergentes e instituyentes de producción de significación, transformación del espacio significante y transformación de las prácticas dialógicas discursivas de significación.

Por tanto, se toma en el presente trabajo, bajo lo desarrollado, como sujeto de estudio para la construcción analítica expuesta a las Organizaciones Civiles Ambientales de la Provincia de San Juan (en adelante OCASJ) que apelan a la construcción crítica como origen del desarrollo de una consciencia sobre el territorio. Presentándose esta crítica, desde las articulaciones dialógicas espaciales locales no legitimadas por las producciones de sentido discursivo público. Convirtiendo estas articulaciones dialógicas discursivas con lo público, las prácticas significantes de estas organizaciones, en construcciones emergentes que buscan en su presencia y dinámica oportunidades de legitimación. Dando origen a prácticas estéticas que presentan, en su sola existencia nuevas significaciones con nuevas capacidades instituyentes.

También se busca, desde esta perspectiva, el análisis de estos los diálogos locales contemporáneos con los nuevos espacios de la globalidad desde la incorporación conceptual de una mirada interlocal.

Contemplando al territorio, como se menciona, desde el "lugar" construido en la caracterización y categorización de los espacios locales y las articulaciones dialógicas implicadas, tomado a los mismos, como elementos significantes del proceso de significación actoral, histórico y local.

Identificando en esta caracterización y categorización, regímenes enunciativos que afectan las formas de articulación dialógica en la producción y reproducción de sentidos discursivos, como también en el reconocimiento de los mismos.

Permitiéndonos el registro de estos regímenes enunciativos, observar la narrativa histórica de las articulaciones espaciales locales, sus transformaciones y cambios.

Esta mirada espacio-territorial de análisis de las condiciones de producción y reproducción del sentido en los discursos, nos permite identificar cualidades de accesibilidad (de 
los actores estudiados) a la producción y reproducción local de sentido discursivo en el entramado de articulaciones dialógicas actorales involucradas.

La cual busca operarse, a través de la identificación organizacional de las estructuras económicas (técnicas, tecnológicas), político/sociales (organizacionales, institucionales) y de conocimiento (lenguaje: formal-académico- e informal-local-), de los miembros de la organización y de la misma como colectivo, como ejes de accesibilidad.

Esto implica la construcción de metodologías de investigación: recolección y análisis de datos, propuestas conceptuales de análisis, etc. desde un entendimiento multifrontal que habilite el necesario desarrollo crítico.

Ejes conceptuales de análisis a desarrollar:

- Relaciones de los procesos de producción de sentido discursivo con las dinámicas espaciales (articulaciones, configuraciones y legitimaciones): prácticas, usos y discursividad implicados.

- Caracterización y categorización de los espacios locales articuladores de la producción y reproducción de sentido discursivo local.

- Regímenes enunciativos y regímenes de visibilidad de los espacios locales implicados en las articulaciones dialógicas de producción y reproducción de sentido discursivo local. - Aproximación a la identificación de los mapas de producción de sentido discursivo local/interlocal de las OCASJ desde su actuación local.

El desarrollo de estos ejes, busca dar respuesta a cuestionamientos como: ¿Cuáles son las huellas semánticas en la producción de sentidos discursivos de las OCASJ que representan relaciones ecosistémicas locales emergentes? Es decir ¿Qué cualidades de accesibilidad de este actor se hallan presentes en los sentidos de sus discursos? ¿Cuál es el grado de conciencia y valoración sobre dicha relación de representación? ¿Qué potencialidades presenta la identificación de estas relaciones sobre las valoraciones y desarrollo de las accesibilidades implicadas?

\section{Categorización y Caracterización del Espacio Local. La Construcción de un Espacio Común}

Primero que nada, es necesario observar la importancia contemporánea de evaluar la implicación del elemento espacial en el proceso de producción de sentidos locales, debido a la multiplicación masiva de las posibilidades de articulación discursiva, favorecida por el desarrollo de los espacios digitales y de sus articulaciones con los tradicionales espacios locales.

Buscando construir categorías conceptuales que nos permitan identificar, caracterizar y categorizar los espacios locales desde las dinámicas de articulación que presentan sus actores. Considerando a dichos espacios como elementos discursivos contextuales a partir de los cuales se generan las producciones y reproducciones del sentido de otros discursos, 
como articulación interdiscursiva original. Analizando las posibilidades de accesibilidad que puedan involucrar cada una de las categorías espaciales identificadas, como elementos incidentes de la participación actoral en la construcción de los procesos ecosistémicos locales.

A partir del desarrollo y construcción de estas categorías se pretende construir una herramienta de análisis de dichos procesos de producción y reproducción de los sentidos discursivos de, en este caso, las OCASJ.

\section{Categorías del Espacio Local:}

- Espacio Público: Son espacios que presentan configuraciones de uso normativas, generadas colectivamente con centro en la convivencia local (sostenimiento del ecosistema local). Su nivel de restricción tiene relación con la densidad de personas que habitan el espacio. Tiene mayor densidad poblacional y mayores configuraciones de tránsito que los espacios privados, dichas configuraciones pueden observarse en las prácticas sociales legitimadas sobre dicho espacio.

- Espacio Privado: Estos espacios presentan mayores posibilidades de modificación de las configuraciones de uso, más disposición del espacio, menor densidad poblacional y menor tránsito. La convivencia, en estos espacios, se encuentra abierta a mayores posibilidades de experimentación y cambio, y posee relaciones de familiaridad. Se hallan en los mismos, mayores posibilidades de modificación particular de este y sus sentidos, que en el espacio público.

- Espacio Común: Nace de la necesidad de visibilizar la existencia de aquellos espacios que buscan articular los sentidos privados y públicos, como es el caso de las OCASJ. Su objetivo es la articulación colectiva de las producciones de sentido particulares/ privadas para la incorporación de estas articulaciones configuradas a la estructura pública institucional. Generalmente se caracterizan por presentarse como espacios de apropiación de los espacios instituidos, es decir como espacios de producción de sentidos emergentes colectivos.

\section{Identificación de los Regímenes Enunciativos Implicados en las Diferentes Categorías del Espacio Local}

Realizaremos un análisis desde una concepción del conocimiento local ${ }^{1}$ basada en las categorías de la experiencia: individual, colectiva y pública en los diferentes espacios locales (privado, público y común) desarrollados para la identificación de los regímenes enunciativos implicados y de su relación con la accesibilidad a la producción y reproducción del sentido local en los discursos.

Tomando a los espacios locales de articulación como referentes de los regímenes de enunciación. 


\section{Experiencia Particular:}

- En Espacios Privados del particular: se presenta un menor condicionamiento social sobre prácticas y usos particulares en dicho espacio y por tanto sobre las producciones y reproducciones particulares de sentido ${ }^{2}$ en estos discursos privados. Dicho condicionamiento debe atender a las referencias institucionales públicas de lo privado particular. En el espacio privado particular no se busca incidir en el espacio público.

Existe una mayor accesibilidad particular a la producción y reproducción de sentido en discursos privados propios.

- En Espacios Privados de otro particular: se presenta un condicionamiento privado de las prácticas y usos particulares sobre dicho espacio y por tanto de las producciones y reproducciones particulares de sentido en sus discursos. Dicho condicionamiento debe atender a las referencias institucionales públicas de lo privado particular.

Existe una menor accesibilidad particular a la producción y reproducción de sentidos en discursos privados de otro particular.

- En Espacios Privados de un actor público: al igual que en los casos anteriores, se presenta un condicionamiento privado de las prácticas y usos particulares sobre dicho espacio y por tanto de las producciones y reproducciones particulares de sentido en sus discursos. Pero dichos condicionamientos deben atender tanto a las referencias institucionales públicas de lo privado con acceso público, en este caso, como a las representaciones sociales de lo privado con acceso público no institucionalizadas. Ya que en este caso si se busca incidir en el espacio público.

Existe una menor accesibilidad particular a la producción y reproducción de sentidos en discursos privados de un actor público.

- En Espacios Públicos: se presenta un mayor condicionamiento social sobre prácticas y usos particulares del espacio y por tanto sobre las producciones y reproducciones particulares de sentido en los discursos públicos. Las posibilidades de incidencia particular en las producciones y reproducciones de sentido en discursos públicos se hallan principalmente en las formas de ejercer las prácticas y usos propuestos por dicho espacio y sus instituciones. Y en la "presencia" que en dicho espacio puedan configurar las nuevas formas de ejercicio de dichas prácticas y usos; como también en la posibilidad de construcción de colectividad a partir de las mismas. Esto implica la posibilidad de construcción de espacios comunes.

Existe una menor accesibilidad particular a la producción y reproducción de sentidos en discursos públicos. Pero podemos observar que existe una relación de accesibilidad "particular" dialógica con dicho espacio público.

- En Espacios Comunes: se presenta una participación particular incidente en los condicionamientos sociales del colectivo que constituye dicho espacio, esto implica a las prácticas y usos particulares y por tanto a las producciones y reproducciones particulares de sentido en discursos comunes. 
Existe una accesibilidad particular consensuada colectivamente respecto a la producción y reproducción de sentidos en discursos comunes.

\section{Experiencia Colectiva:}

- En Espacios Privados de un particular o de un actor público: existe un condicionamiento privado y particular de las prácticas y usos colectivos sobre dicho espacio, y por tanto de las producciones y reproducciones colectivas de sentido en sus discursos privados. Dicho condicionamiento debe atender a las referencias institucionales públicas de lo privado.

Existe una menor accesibilidad colectiva a la producción y reproducción de sentidos en discursos privados.

- En Espacios Públicos: existe un condicionamiento social representado en el espacio público y sus instituciones sobre las prácticas y usos colectivos de dicho espacio y por tanto sobre las producciones y reproducciones colectivas de sentido en discursos públicos. Existe una accesibilidad colectiva dialógica con el espacio público respecto a las producciones y reproducciones de sentidos en los discursos públicos

Esto quiere decir que, las formas de las prácticas y usos colectivos en el espacio público pueden concordar con dichos condicionamientos que el mismo representa o pueden generar nuevas propuestas en las formas de ejercer prácticas y usos. Exhibiendo la posibilidad dialógica mencionada, que implica una relación dialógica entre lo instituido y lo emergente ${ }^{3}$.

- En Espacios Comunes: existe una participación colectiva incidente en la construcción de estos espacios y los condicionamientos de sus propias prácticas y usos; y por tanto sobre las producciones y reproducciones colectivas de sentidos en los discursos comunes.

Es decir que: Estos espacios se crean a partir de las experiencias colectivas de prácticas y usos de apropiación de los espacios públicos y privados de actores públicos, por tanto, se genera una construcción emergente de sentido en los discursos comunes propios de estas prácticas.

Estas construcciones emergentes buscan su legitimación en el espacio público.

\section{Experiencia Pública:}

- En Espacios Privados: la experiencia pública en espacios privados implica al discurso público, que se reproduce a través de espacios técnico-tecnológicos de mediación. Lo que dificulta el diálogo, ya que dicho diálogo queda condicionado a las posibilidades técnicas y tecnológicas, político/sociales y de conocimiento (lenguaje) del medio; o queda condicionado al acceso particular, tanto al medio como al espacio público y sus instituciones, a los que refiere el discurso. Es decir, a la experiencia a través de prácticas y usos particulares de dicho espacio público. Este diálogo tiene una relación de representación directa de las accesibilidades públicas y privadas implicadas (incluyendo la de los medios). Convirtiéndo- 
se, esta experiencia del discurso público, en una triada dialógica entre las representaciones del espacio público, los medios y las experiencias públicas de los particulares.

- En el Espacio Público: la experiencia pública (discursos, normativa, etc) en el espacio público, se halla condicionada por las propias prácticas y usos que habilita dicho espacio público y por tanto por las producciones y reproducciones de sentido legitimadas en el mismo y sus instituciones, como representación de la narrativa histórica de hechos que lo caracterizan. Es decir, como representación de la narrativa histórica de diálogos entre los espacios, experiencias, sentidos y discursos locales, y como representación de la dinámica de relación entre los mismos. Dinámica que presenta un carácter procesual que debe respetarse o acrecentarse para mantener dicha experiencia su carácter de pública. Refiriéndonos con dicha dinámica, a las relaciones ecosistémicas entre los espacios y sentidos locales implicados, y a las posibilidades de crecimiento de las mismas.

- En Espacios Comunes: Debido a que los espacios comunes nacen de prácticas y usos colectivos de apropiación de los espacios públicos y privados de actores públicos, en busca de una legitimación pública de dichas apropiaciones. La experiencia pública, en estos espacios comunes, constituye una instancia referente de gestión de los sentidos a través de las formas. Es decir, una instancia referente en cuanto a las formas, tipos y dinámicas de articulación de los discursos públicos (regímenes de visibilidad). Ya que al igual que en el caso de la experiencia pública en espacios públicos, estos últimos cumplían un rol de referencia por las significaciones mencionadas implicadas. De la misma manera, en este caso, la experiencia de lo público constituye una referencia para los espacios comunes en las articulaciones y relaciones que deberán respetar o acrecentar para mantener su carácter común, es decir, para generar una dinámica plural y no una institución desarticulada de ordenes alternativos.

En estas categorías, basadas en una concepción de conocimiento local articulada con las categorías del espacio local construidas en el presente trabajo; se generan, en forma paralela, lo que podemos identificar como "regímenes enunciativos locales". Pudiendo, en base a lo desarrollado, categorizarlos y caracterizarlos de la siguiente manera:

- Regímenes enunciativos privados: se producen en espacios privados y nos permiten identificar aquellos discursos categorizados como privados. Se caracterizan por el tipo de relación que se guarda con el espacio: si el espacio privado es propio, de otro particular o de un actor público. Ya que será aquel actor que presente pertenencia sobre el mismo, el que determine las condiciones de dicho espacio y de quienes lo habitan o puedan habitarlo. Si bien, estas condiciones, siempre se encontrarán dentro de las legitimaciones públicas de los espacios privados, las cuales determinarán su caracterización y posibles categorizaciones e implicancias públicas como institución; este es el único diálogo obligatorio determinado. Estas características, se reflejan en los discursos privados y sus categorizaciones como particulares o de acceso público que representan el tipo de accesibilidad a la producción y reproducción de dichos regímenes. 
- Regímenes enunciativos públicos: se producen en los espacios públicos y nos permiten identificar aquellos discursos categorizados como públicos. Se caracterizan por la relación dialógica (articuladora) implicada en las experiencias particulares, colectivas e institucionales (públicas) con dicho espacio. Estas características se reflejan en los discursos públicos categorizados como particulares, colectivos o institucionales (públicos) por el tipo de accesibilidad a la producción y reproducción de dichos regímenes.

- Regímenes enunciativos comunes: se producen en espacios comunes y nos permiten identificar aquellos discursos categorizados como comunes. Se caracterizan por la participación incidente particular y colectiva en los condicionamientos del colectivo que constituye dicho espacio común, y su búsqueda de legitimación pública. Estas características se reflejan en los discursos comunes categorizados como particulares o colectivos (organizacionales). La accesibilidad a la producción y reproducción de dichos regímenes queda determinada por la presencia participativa en la construcción de los mismos.

\section{Procesos de Significación Local y Su Relación con la Globalidad}

El análisis planteado desde la articulación de las prácticas, sentidos y discursos con los espacios locales como dimensión significante dentro de concepciones como la de lugar y territorio, genera algunos cuestionamientos respecto a prácticas contemporáneas relacionadas con dichos espacios como ¿Qué lugar ocupan dentro del desarrollo descripto los espacios virtuales digitales (que antes hemos mencionado)?

Buscando dar respuesta a esta duda, es necesario recordar la articulación que estos nuevos espacios presentan con concepciones masificadas como la de "globalización". Ya que esta concepción, nos direccionará hacia otras preguntas relacionadas como ¿De qué forma estos espacios locales se articulan con dicha idea de globalidad?

Dispuestos a sostener una perspectiva basada en estos lugares territorializados, nos adentraremos en una concepción de la globalización.

Comenzaremos por enunciar su origen económico que, según la reflexión de autores como Arif Dirlik (1997) citado en Escobar Arturo (2000) ha permitido igualar dicha concepción al capital, su relación con el espacio, la historia y sus prácticas. Generando una división con lo local que, desde su forma polarizada define como, las relaciones con el trabajo y las tradiciones, dejando afuera la construcción de puntos de articulación entre estas partes.

Es precisamente esta, una de las tareas a las cuales buscaremos aproximarnos a través de nuestro aporte. Comenzando por descomponer las posibles relaciones que se puedan plantear estos "lugares distintos". Tratando de encontrar en dichos puntos de articulación la generación de categorías conceptuales analíticas que nos permitan observar, identificar y analizar los mismos y sus estructuras de significación.

Habiendo podido dividir los espacios de significación local en privados, públicos y en una construcción de lo común, para la observación de los mismos como base de regímenes enunciativos representativos de formas de accesibilidad a la producción y reproducción de sentidos discursivos locales ¿Podemos entender a esta globalización y a los espacios 
virtuales, causantes o consecuentes de la misma, involucrados en estos procesos locales de significación dados en dicha articulación espacial? Y siendo así ¿Podemos identificar las estructuras y organizaciones de estas articulaciones con lo "no local"?

El primer paso que tomaremos en esta dirección, y que enunciaremos en el presente artículo, será el de identificar y analizar conceptualmente las etapas que involucran al proceso productivo de sentidos discursivos, si bien ya lo venimos enunciando, buscaremos desarrollar conceptualmente los análisis implicados, en la toma de decisión de esta división categórica.

Pudiendo identificar, una primera etapa productiva que consiste en el desarrollo de hechos que dan significación a los elementos contextuales significantes en un proceso de producción de sentidos y discursos, entendiendo a estos últimos desde una relación estratégica con dichos sentidos en su articulación con los regímenes enunciativos propios de los espacios discursivos, que es lo que se viene desarrollando.

Aquí, dichos discursos, no solo se presentan como instancia ultima de dicha etapa de producción, sino que se presentan como primera instancia de una segunda etapa: la de reproducción de los sentidos implicados en la articulación discursiva de la producción mencionada. Siendo esta etapa el objetivo del componente estratégico-productivo de los discursos.

Observando también que, los regímenes enunciativos desarrollados, afectan no solo a dicha etapa productiva, sino también a esta etapa reproductiva desde la representación del posicionamiento ecosistémico local significante, en cuanto a las formas de accesibilidad a la construcción de sentido local.

Considerando importante, en este punto, hacer mención a nuestra referencia a la concepción de "sistema productivo" de Eliseo Verón, con la cual el mismo refiere a la "articulación (...) entre producción, circulación y consumo de conocimiento" (1993, p. 16).

Desarrollando, en este caso, por su relación con nuestra segunda etapa, su eje de circulación: en el cual describe como "las condiciones de constitución del campo de efectos de sentido varían según la naturaleza de la circulación; en otras palabras, según el tipo de intercambio significante de que se trata" (Verón, 1993, p. 16).

Esta afirmación, en el presente trabajo, se interpreta que nos habla de la importancia de las articulaciones discursivas y del espacio situado en que se desarrollan, como elemento significante, lo cual nos direcciona al desarrollo de los ejes descriptos en la presente investigación. En otras palabras, interpretamos que refiere a las experiencias como escenarios interdiscursivos de significación.

Esta similitud en la valoración de la experiencia como elemento de significación, redimensiona la importancia de la construcción conceptual de los espacios locales y sus consecuentes regímenes enunciativos como bases significantes.

Por lo tanto, retomando y ya habiendo dado mención a nuestra segunda etapa de las condiciones de producción: "la reproducción"; nos adentraremos en el desarrollo de su conceptualización para, desde su descomposición habilitar el análisis de las condiciones de reproducción de nuestro sujeto de estudio.

Observando, que las condiciones de reproducción refieren a elementos contextuales significantes articulados con el cumplimiento de los objetivos de reproducción de sentidos a los que aspiran los discursos. 
Es aquí, que deberemos analizar cómo afectan este proceso de significación los espacios locales donde se reproducen estos discursos y a que regímenes enunciativos locales responden los mismos.

Observando que, la lectura de estas formas de accesibilidad de origen a la construcción del sentido local, es decir, la lectura de los regímenes enunciativos implicados en los discursos, dependerá de la relación con el conocimiento local de origen. Dándose esta lectura, principalmente, sobre la aparición implícita de este conocimiento local en las huellas semánticas que aparecen en estas reproducciones y en la generación de los regímenes de visibilidad.

\section{Conclusión}

La posibilidad de identificación de los ejes de accesibilidad económico (técnico, tecnológico), político/social (organizacional, institucional) y de conocimiento (lenguaje: formal -académico- e informal -local-) de las OCASJ nos permitirá introducirnos en la observación de la construcción local de los mismos. Presentando, esta identificación, una posibilidad de desarrollo de pensamientos críticos y conscientes sobre dichos ejes locales.

Permitiendo esto, el planteo de debates sobre concepciones dependientes de estas relaciones que describen entre la comunidad y su entorno. Como lo son las concepciones sobre el propio entorno natural y la naturaleza presentes en las construcciones de sentido y discurso que dan origen a estas organizaciones; como también concepciones como las de "crecimiento" o "progreso". Habilitando, este proceso, la identificación de dichas construcciones conceptuales, en las particularidades culturales de las relaciones locales y sus valoraciones, representadas en los espacios de articulación local como construcciones significantes de posibilidad de transformación y cambio.

Esta propuesta conceptual busca generar una perspectiva de construcción de dinámicas plurales como ciclos retroalimentados que, si bien puedan comenzar en el objetivo de institución pública de articulaciones espaciales y sentidos emergentes, proyecte que estas concreciones contemplen la restitución de dichos procesos instituyentes a nuevas articulaciones de sentido y discurso. Para lo cual, dichas propuestas de sentidos emergentes que se instituyan como públicas, deberán buscar legitimar la construcción de espacios locales de diálogo.

Desde aquí es que se trabaja la construcción conceptual de una categoría de espacio de lo común que busca contemplar esta necesidad, surgida de la observación de los "lugares" de articulación que se generan en el territorio entre los espacios públicos y privados, que se conciben como elementos significantes dentro de los mencionados procesos de producción de sentido y discurso local. Denominando a esta construcción categórica conceptual de espacio: espacio común. 


\section{Notas}

1. Sobre el cual Escobar Arturo cuestiona, en su desarrollo teórico"...de si 'conocimiento local' es, en sí, una etiqueta apropiada para los mecanismos cognitivos y experimentales que están en juego en las relaciones de la gente con los entornos no humanos ..." (2000, p. 8).

2. Se considera a las prácticas y usos sobre el espacio elementos de producción y reproducción de sentidos, convirtiendo esto a las mismas en elementos discursivos.

3. Podemos observar el desarrollo de estos conceptos sobre lo instituido y lo instituyente en el texto de Castoriadis, C. (2006). Y sobre el desarrollo aplicado de lo emergente en el trabajo de tesis de Racioppe, B. (2014).

\section{Referencias Bibliográficas}

Castoriadis, C. (2006). Figuras de lo Pensable México: Editorial del Fondo de la Cultura Económica.

Cigolani, G. y Fernández, M. (2010). Oficios terrestres. Televisión y política: espacio público, puestas en escena y regímenes de visibilidad, 16 (25), 37-49.

Coraggio, J. L. (2011). Economía social y solidaria. El trabajo antes que el capital Quito: Ediciones Abya Yala.

Dirlik, A. (2000). Place-based Imagination: Globalism and the Politics of Place. Nueva York. Citado en: Escobar, A. (2000). El lugar de la naturaleza y la naturaleza del lugar: ¿Globalización o Posdesarrollo? En: La colonialidad del saber: eurocentrismo y ciencias sociales. Perspectivas Latinoamericanas (pp. 246-287) Buenos Aires: CLACSO, Consejo Latinoamericano de Ciencias Sociales.

Escobar, A. (2000). El lugar de la naturaleza y la naturaleza del lugar: ¿Globalización o Posdesarrollo? En: La colonialidad del saber: eurocentrismo y ciencias sociales. Perspectivas Latinoamericanas (pp. 246-287) Buenos Aires: CLACSO, Consejo Latinoamericano de Ciencias Sociales.

Espoz, M. (Ed.) (2017). Sentires (in)visibles. La construcción de entornos en espacios socio segregados Buenos Aires: Teseo Press.

Gudynas, E. (2004). Ecología, economía y ética del desarrollo sostenible Montevideo: Coscoroba Ediciones.

Leff, E. (2005). OSAL. La geopolítica de la biodiversidad y el desarrollo sustentable. Economización del mundo, racionalidad ambiental y reapropiación social de la naturaleza, 6 (17), 263-273.

Racioppe, B. (2014). Cultura libre y copyleft: hacia una redefinición en la manera de entender la producción artística La Plata: UNLP. Disponible en: http://sedici.unlp.edu.ar/ handle/10915/44651

Verón, E. (1993). La semiosis social. Fragmentos de una teoría de la discursividad. Barcelona: Editorial Gedisa. 


\begin{abstract}
Interesting in highlighting the historical characterization of art as a disruptive political element. And it is, observing that this process has only been possible from a conscious and critical articulation of the local spaces and their characterizations, that the present work, is situated in the search of alternative productions to the established orders. Seeing its legitimacy in the representation of the joints denied by the local space. This objective is worked, through the analysis of the productions of sense of the Civil Environmental Organizations of the Province of San Juan, understanding said actor as representative of these articulations. Being able to identify in their visibility regimes, a reproductive stage that raises in the very existence of these organizations and in their processes of reproduction of senses an economic, aesthetic and local alternative political proposal, that is, a conceptual proposal that considers a plural order, as a representation of the need to establish new types of space that make visible the intrinsic articulation between the public and the private given in the integral process of production of local meaning. As an emergent order, based on an ecosystemic perspective of the articulated local, in an interlocal form, within the logics of the global organism.
\end{abstract}

Keywords: Local - Ecosystem - Visibility - Aesthetics - Communication.

Resumo: Interessados em destacar a caracterização histórica da arte como elemento político disruptivo. E é, observando que o processo só foi possível a partir de uma consciente e crítica de áreas locais e suas caracterizações conjuntas, este trabalho está em encontrar alternativas para produções ordens estabelecidas. Vendo sua legitimidade na representação das articulações negadas pelo espaço local. Este objetivo é trabalhado, através da análise das produções de sentido das Organizações Ambientais Civis da Província de San Juan, entendendo dito ator como representativo dessas articulações. Ser capaz de identificar regimes de visibilidade, a fase reprodutiva aumenta a própria existência dessas organizações e seus processos de reprodução sente uma proposta econômica, alternativa local, estética e política, ou seja, uma proposta conceitual que considera uma ordem plural, como representando a necessidade de novos tipos de espaço que ilustram a articulação intrínseca entre o público e o privado dado no sentido local integral processo de produção. Como uma ordem emergente, baseada em uma perspectiva ecossistêmica do local articulado, em uma forma interlocal, dentro das lógicas do organismo global.

Palavras chave: Local - Ecossistema - Visibilidade - Estética - Comunicação.

[Las traducciones de los abstracts fueron supervisadas por el autor de cada artículo] 